\title{
Effect of Magnetic Stirring on the Green Synthesis of Silver Nanoparticles Using Lavandula angustifolia Plant Extract
}

\author{
M. Villalpando ${ }^{1 *}$ and G. Rosas ${ }^{1}$ \\ 1. Instituto de Investigaciones en Metalurgia y Materiales, Departamento de Metalurgia física, UMSNH, \\ Morelia, México. \\ * Corresponding author: mvillalpando@umich.mx
}

Currently, the synthesis of silver nanostructures, especially nanoparticles (NPs) has increased considerably due to their high demand in various consumer products such as textiles, medical products, paints, cosmetics, packaging of food, among others [1]. For this reason, green synthesis is considered a viable alternative because it reduces the use and generation of toxic reagents for the environment and human health [2]. However, controlling the polydispersity of NPs is a significant challenge that can be improved with variations in the experimental parameters [3]. Thus, the present work evaluates the effect of the magnetic stirring on the formation of silver nanoparticles (AgNPs) using a green approach by the use of Lavandula angustifolia plant extract as a reducing and stabilizing agent.

Lavandula angustifolia was mechanically triturated in a ball mill to obtain fine powders. The infusion was done mixing $2 \mathrm{~g}$ in $100 \mathrm{ml}$ of deionized water $(0.02 \mathrm{~g} / \mathrm{ml})$. The solution was heating at $60{ }^{\circ} \mathrm{C}$ for 20 min and finally was filtered. Also, silver nitrate salt precursor $\left(\mathrm{AgNO}_{3}\right)$ was dissolved in deionized water $(10 \mathrm{mM})$. The synthesis reaction consisted of mix plant extract and $\mathrm{AgNO}_{3}$ solution at room temperature with a volumetric relation 1:1 varying magnetic stirring $(0,200$ and $600 \mathrm{rpm})$ for $24 \mathrm{~h}$. The first indication of silver nanostructures formation was a color change in the solution from yellow to dark brown, black and gray as the stirring magnetic increase (inset Fig. 1). The color change is due to the surface plasmon resonance (SPR) found in the AgNPs. Subsequently, AgNPs were characterized by UV-Vis spectroscopy (Ocean Optics USB-4000). Scanning Electron Microscopy was carried out in a Jeol JSM-7600F microscope. X-ray diffraction in a D8 Advance DAVINCI lynx eye. Finally, Transmission electron microscopy was performed in a Philips Tecnai F20. Fig 1 shows the UV-Vis spectra that confirms the green synthesis reaction. It is observable three spectrums with a characteristic wavelength between 400-550 $\mathrm{nm}$ that it is associated with AgNPs formation. Also, it is appreciated two SPR peaks related to a second size distribution that disappears with an increase in the stirring. For the solution evaluated at $600 \mathrm{rpm}$ the width of the band was greater in comparison with the others, attributed to the formation of larger silver nanostructures. SEM analysis is shown in Fig. 2 demonstrates the presence of silver nanostructures in the reaction medium. Fig. 2a corresponding to the sample without magnetic agitation reaffirms the synthesis of AgNPs. In this case, is observed the aggregation of NPs in small zones forming islands. Otherwise, the sample with slow agitation $(200 \mathrm{rpm})$ presented in Fig. 2b illustrates the separation of the islands improving the distribution. In the case of Fig. 2c it was identified that increasing the magnetic stirring to $600 \mathrm{rpm}$, the number of collisions between nanoparticles increased causing their coalescence, for this reason, the surfactants presented a rearrangement of the initial stabilization that it was reflected in the formation of 1D silver nanostructures as rods. Also, the EDS analysis confirms the presence of reduced silver (Fig 2d). Finally, Fig. 3 display the study of structure, morphology, and size of the better sample $(200 \mathrm{rpm})$ by TEM and XRD. Figure 3a shows that AgNPs obtained are below $100 \mathrm{~nm}$ with an average size of $20 \mathrm{~nm}$ and two morphologies: spherical and triangular. Fig 3b shows a high-resolution image (HR-TEM), and its corresponding FFT pattern reveals the crystalline nature of AgNPs. The lattice fringes correspond to $0.24 \mathrm{~nm}$ distance that belongs to (111) 
crystallographic planes of silver. Fig 3c shows the XRD pattern illustrating the diffraction peaks indexed by (111), (200), (220), (311), and (222) according to the standard diffraction data of silver (No. JCPDS 04-0850) with a lattice parameter of $0.408 \mathrm{~nm}$. In summary, it was evaluated the effect of magnetic stirring in the green synthesis of AgNPs using an aqueous extract of Lavandula angustifolia. The velocity of $200 \mathrm{rpm}$ reflected control in a dispersion of them with an average size of $20 \mathrm{~nm}$ with spherical and triangular morphologies. TEM and XRD corroborate the crystalline structure of nanosolids synthesized.

\section{References}

[1] E McGillicuddy et al., Science of The Total Environment 575 (2017), p. 231.

[2] N Noah. Chapter 6 - Green synthesis: Characterization and application of silver and gold nanoparticles. (2019). Elsevier.

[3] TA Jorge de Souza, LR Rosa Souza and LP Franchi, Ecotoxicology and Environmental Safety 171 (2019), p. 691.

[4] The authors acknowledge funding from Consejo Nacional de Ciencia y Tecnologia (CONACyT), México.

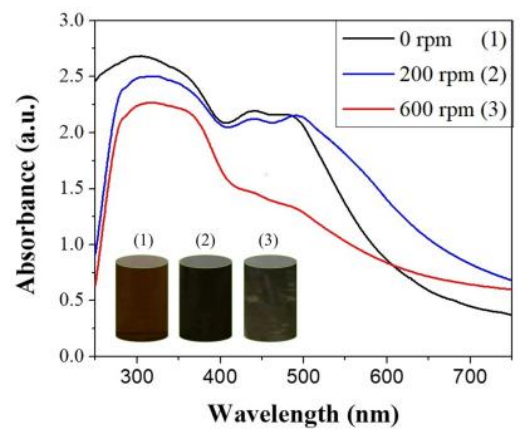

Fig. 1 UV-Vis spectra of AgNPs synthesized with different magnetic stirring.

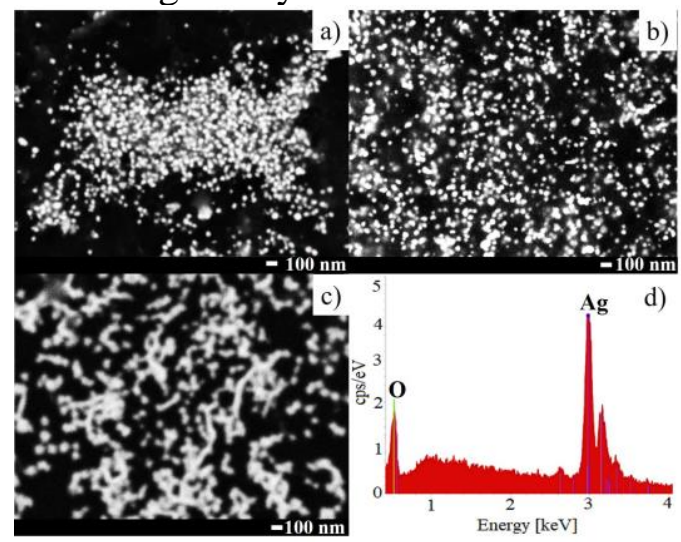

Fig.2 SEM micrographs of silver nanostructures; a) $0 \mathrm{rpm}$, b) $200 \mathrm{rpm}$, c) $600 \mathrm{rpm}$ and d) EDS analysis.
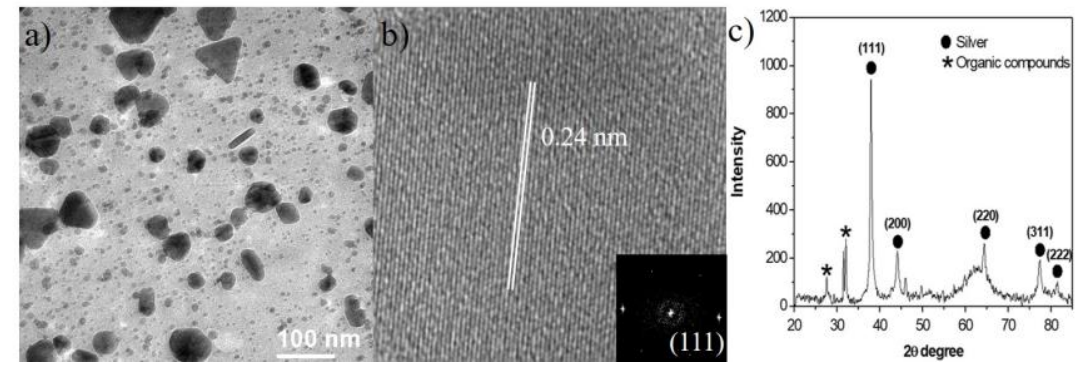

Fig.3 AgNPs synthesized at $200 \mathrm{rpm}$, a) Bright-field image, b) high-resolution image indicating their crystalline nature and c) XRD pattern. 